\title{
O uso do brinquedo terapêutico na administração por inalação em pré-escolares
}

\author{
Using therapeutic toy for administering by inhalation in pre-schools \\ El uso de juguete terapéutico para administración por inhalación en preescolares
}

\author{
Maria Paula Custódio Silva', Mariane Santos Belisário", Nylze Helena Guillarducci Rocha'I", \\ Mariana Torreglosa Ruiz'v , Jesislei Bonolo do Amaral Rochav , Divanice Contim ${ }^{\text {VI }}$
}

\begin{abstract}
RESUMO
Objetivo: comparar o comportamento de pré-escolares durante o uso de administração por inalação, antes e após sessão de Brinquedo Terapêutico. Método: estudo quase-experimental de abordagem quantitativa, com a técnica de observação antes e após a intervenção, realizado por meio de amostra intencional, recrutadas em uma Unidade de Pronto Atendimento no interior de Minas Gerais. Os dados foram analisados por estatística descritiva e inferencial, coletados entre os meses de novembro de 2017 a abril de 2018. Resultados: foram avaliados 25 comportamentos de 99 pré-escolares durante a administração por inalação. Após a sessão, 73,7\% estavam com a postura e expressão facial relaxada, 76,8\% estavam à vontade, 19,2\% interromperam o procedimento e 38,4 \% solicitaram a presença da mãe ou acompanhante. Conclusão: o uso do brinquedo, favoreceu maior aceitação e adaptação de pré-escolares submetidos à administração por inalação, evidenciado a importância em implementar essa estratégia em serviços de pronto atendimento pediátrico.
\end{abstract}

Descritores: Pré-Escolar; Jogos e Brinquedos; Administração por Inalação; Cuidados de Enfermagem.

\begin{abstract}
Objective: to compare the behavior of preschoolers during inhalation therapy, before and after a Therapeutic Play session. Method: quasi-experimental, quantitative study using pre- and post-intervention observation with an intentional sample recruited at an Emergency Care Unit in Minas Gerais. Data were collected from November 2017 to April 2018 and analyzed by descriptive and inferential statistics. Results: twenty-five behaviors of 99 preschoolers were evaluated during inhalation administration. After the session, $73.7 \%$ were relaxed in posture and facial expression, $76.8 \%$ were comfortable, $19.2 \%$ interrupted the procedure, and $38.4 \%$ requested the mother or companion to be present. Conclusion: the use of toys favored greater acceptance and adaptation by preschoolers undergoing inhalation therapy, evidencing the importance of implementing this strategy in pediatric emergency services.
\end{abstract}

Descriptors: Child, Preschool; Play and Playthings; Administration, Inhalation; Nursing Care.

\section{RESUMEN}

Objetivo: comparar el comportamiento de los preescolares durante la terapia de inhalación, antes y después de una sesión de Juego Terapéutico. Método: estudio cuantitativo cuasi-experimental utilizando observación previa y posterior a la intervención con una muestra intencional reclutada en una Unidad de Atención de Emergencia en Minas Gerais. Los datos se recopilaron de noviembre de 2017 a abril de 2018 y se analizaron mediante estadísticas descriptivas e inferenciales. Resultados: se evaluaron veinticinco comportamientos de 99 niños en edad preescolar durante la administración por inhalación. Después de la sesión, el $73.7 \%$ se relajó en la postura y la expresión facial, el $76.8 \%$ se sintió cómodo, el $19.2 \%$ interrumpió el procedimiento y el $38.4 \%$ solicitó que la madre o la acompañante estuvieran presentes. Conclusión: el uso de juguetes favoreció una mayor aceptación y adaptación por parte de los preescolares sometidos a terapia de inhalación, lo que evidencia la importancia de implementar esta estrategia en los servicios de emergencia pediátricos.

Descriptores: Preescolar; Juego e Implementos de Juego; Administración por Inhalación. Atención de Enfermería.

\section{INTRODUÇÃO}

A palavra brincar demonstra o quanto a atividade lúdica é essencial para o desenvolvimento infantil, ou seja, é brincando que, desde cedo, a criança se integra a si mesma, às outras pessoas e ao meio ambiente. É a forma mais genuína utilizada em diferentes contextos assistenciais capaz de atenuar a expressão de sentimentos, como ansiedades e frustrações advindos de procedimentos ou situações desagradáveis e dolorosas, o que de outra forma não seria possível, em função da sua imaturidade emocional ${ }^{1,2}$

'Enfermeira. Mestre. Aluna do curso de doutorado. Universidade Federal do Triângulo Mineiro. Uberaba, Brasil. E-mail: maria_paulacs@hotmail.com. ORCID: https://orcid.org/0000-0001-8694-1589.

"Enfermeira. Especialista. Universidade Federal do Triângulo Mineiro. Uberaba, Brasil. E-mail: mariane.belisario@yahoo.com.br. ORCID: https://orcid.org/0000-0003-35666171.

I'Enfermeira. Especialista. Aluna do curso de mestrado. Universidade Federal do Triângulo Mineiro. Uberaba, Brasil. E-mail: nylze@hotmail.com. ORCID: https://orcid.org/0000-0002-6615-1667.

IVEnfermeira. Doutora. Professora Adjunta. Universidade Federal do Triângulo Mineiro. Uberaba, Brasil. E-mail: marianatorreglosa@hotmail.com. ORCID: https://orcid.org/0000-0002-5199-7328.

VEnfermeira. Doutora. Professora Adjunta. Universidade Federal do Triângulo Mineiro. Uberaba, Brasil. E-mail: jesisleiamaralrocha@gmail.com. ORCID: https://orcid.org/0000-0002-0591-7972.

v'Enfermeira. Doutora. Professora Associada. Universidade Federal do Triângulo Mineiro. Uberaba, Brasil. E-mail: d.contim@uol.com.br. ORCID: https://orcid.org/0000-00015213-1465. 
O Brinquedo Terapêutico (BT) é uma atividade estruturada, aplicada por um profissional capacitado, cuja finalidade é promover o bem-estar físico e emocional da criança ao vivenciar situações pouco familiares a sua idade, como compreender o momento da internação e sua doença naquele momento ${ }^{3}$. Essa atividade facilita a interação profissional-criança, favorecendo a realização dos procedimentos de forma menos dolorosa e mais humanizada, amenizando sentimentos de revolta, depressão e tristeza advindos da associação que a criança faz da hospitalização a um castigo ou punição ${ }^{4}$.

Ao incorporar o BT durante a prestação do cuidado, o enfermeiro procura estabelecer a comunicação e relacionamento com a criança, essas estratégias lúdicas facilitam a interação e melhoram a qualidade da assistência. É competência do enfermeiro a aplicação do BT no contexto da assistência à criança, porém enfermeiros ainda encontram dificuldades em aplicá-la, destacando como principais motivos a falta de tempo, despreparo e a falta de recursos materiais $^{6,7}$.

A escolha por doenças respiratórias agudas deve-se ao fato dessas constituírem um grupo importante na população infantil, descritas como a causa mais frequente de mortalidade infantil nos países em desenvolvimento, atingindo principalmente crianças menores de cinco anos de idade, sendo as Unidades Pronto Atendimentos (UPAS) a porta de entrada destas doenças no Sistema Único de Saúde. Dentre as doenças respiratórias agudas, as Infecções Respiratórias Agudas (IRA) são as causas mais comuns de morbimortalidade na infância ${ }^{8,9}$. A principal forma de tratamento para IRA é a administração por inalação, o uso adequado da técnica tem sido fator responsável pelo controle da doença ${ }^{10,11}$. O método tem várias desvantagens que aumentam a ansiedade e desconforto da criança como o uso da máscara e o barulho do aparelho ou barulho da rede de oxigênio ${ }^{12}$.

\section{REVISÃO DE LITERATURA}

Estudos sobre a aplicação do BT por enfermeiros têm se destacado com a população infantil ${ }^{13-18}$. Evidenciam o uso do lúdico para minimizar o desgaste provocado por agravos como o câncer, diabetes e outras doenças crônica ${ }^{13-15}$, e no preparo para procedimentos como punção venosa, administração de medicamentos, vacinas e procedimentos cirúrgicos ${ }^{16-18}$. Tornando estratégia facilitadora na interação da tríade família/criança/profissionais da saúde durante a assistência de enfermagem ${ }^{1,2}$.

Nos espaços da emergência pediátrica o BT tem promovido benefícios quanto a integralidade na atenção ao estado de saúde e melhor aceitação dos procedimentos necessários ao diagnóstico e tratamento ${ }^{2,19}$. Estudo sobre o uso do BT em crianças pré-escolares durante a administração por inalação indicou que estas completaram o tratamento, aumentando a satisfação ${ }^{12}$.

Desse modo, verifica-se a necessidade de novas pesquisas, para que se evidencie, neste e em outros contextos, a percepção da equipe de Enfermagem. Nessa direção objetivou-se comparar o comportamento de pré-escolares durante o uso da administração por inalação, antes e após a sessão de BT.

\section{MÉTOdo}

Trata-se de um estudo quase-experimental, de abordagem quantitativa, com a técnica de observação antes e após a intervenção. Os quase-experimentos carecem de distribuição aleatória como manipulação de uma variável independente, porém possui as características de randomização e/ou grupo controle ${ }^{20}$. Nesse estudo, considerou-se como variável independente a manipulação do brinquedo no preparo antes do procedimento e, dependente, as reações após o uso do BT por pré-escolares. O cenário do estudo foi uma UPA situada no interior do estado de Minas Gerais. A coleta de dados foi realizada entre os meses de novembro de 2017 a abril de 2018.

A população da pesquisa foi selecionada a partir do cálculo sobre o atendimento de pré-escolares com necessidade de administração por inalação, entre os meses janeiro/junho de 2017 no cenário do estudo. Nesse período foram realizados 888 atendimentos. Desta forma, a intenção foi de realizar o estudo com 10\% da população atendida, resultando em número amostral de aproximadamente 88 crianças.

As crianças incluídas estavam na faixa etária da pré-escola (três a cinco anos e 11 meses), segundo critérios do Ministério da Saúde do Brasil²; não tinham comprometimento cognitivo e receberam duas ou mais sessões de administração por inalação no mesmo atendimento e estavam em condições de brincar, ou seja, não apresentavam estado geral grave e estavam consciente e contactuando com o meio ambiente. Optou-se por excluir as que não estavam acompanhadas por pai e/ou mãe no momento do procedimento.

A coleta de dados foi executada em quatro etapas. Na primeira foi realizada a seleção dos pré-escolares, intencional e auxiliada pela equipe de saúde da UPA, que indicava possíveis participantes conforme os critérios supracitados. Por meio do prontuário, buscou-se à idade, nome dos pais, e estado geral da criança. Os pais dos préescolares foram abordados e convidados a participarem do estudo, mediante assinatura do Termo de Consentimento 
Livre e Esclarecido, resguardando, contudo, o direito de negar a participação no estudo. Todos abordados aceitaram participar.

Na segunda etapa, foram observados os comportamentos dos pré-escolares durante a realização da primeira administração por inalação. Essa etapa serviu para tria-los conforme critérios de inclusão estabelecidos.

Já na terceira etapa efetuou-se uma entrevista com um dos pais para obtenção dos dados de caracterização da criança, questionando gênero e idade, se frequenta creche ou escola, procedência, quem cuida da criança no domicilio, doença que levou a criança à UPA e se a criança já esteve internada, complementando assim os dados colhidos do prontuário. Nessa etapa a criança foi abordada e convidada a participar da sessão de BT e uma das pesquisadoras demonstrava o procedimento na boneca, solicitando a participação da criança que, ao final da dramatização, era convidada a repetir a brincadeira, 12 recusaram participar e 15 iniciaram a brincadeira e não foram até o final devido a não querer brincar ou piora da condição clínica, registrando 23 exclusões. Na quarta etapa criança foi submetida a segunda administração por inalação e foram observados novamente os comportamentos.

Para avaliar a reação dos pré-escolares, foi elaborado um check-list com base em estudos que focalizavam a punção venosa, administração de vacinas e o preparo cirúrgico para avaliação de comportamentos por meio do $\mathrm{BT}^{18,19,20}$. Foi realizada a validação de face e conteúdo deste instrumento, por três juízes, com experiência na área da assistência a criança. O instrumento inicial continha 20 comportamentos, com avaliação dos juízes quatro questões foram complementadas e cinco acrescentadas relacionadas a administração por inalação.

O check-list foi composto por duas colunas: a primeira para transcrição dos comportamentos apresentados durante a primeira administração por inalação e a segunda para anotar os comportamentos identificados após a intervenção do BT. Foram incluídos 25 comportamentos associados à aceitação do procedimento. Na sessão de BT, utilizou-se bonecas e um inalador, feitos com sucata, a partir de garrafas pets de $500 \mathrm{ml}$ transparentes e decorado com motivos infantis (Figura 1). Ressalta-se que duas pesquisadoras observaram independentemente os comportamentos apresentados a fim de avaliar divergências e o tempo médio de cada sessão foi de 20 minutos.

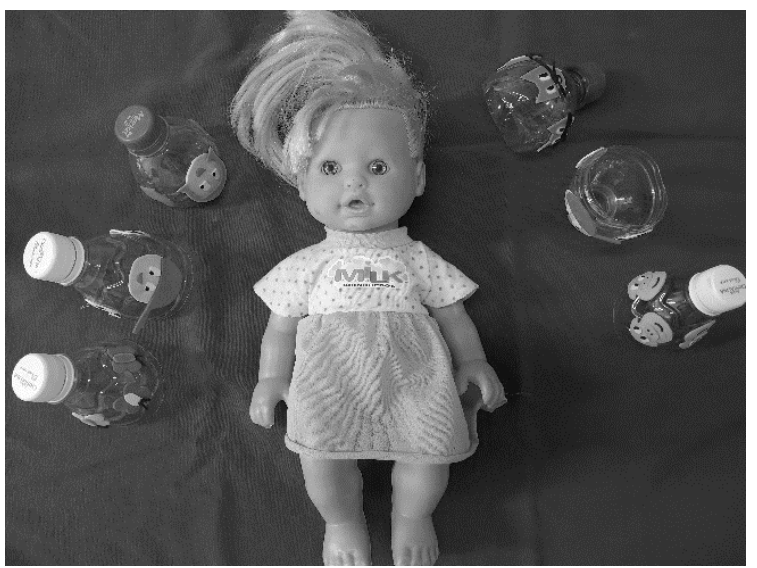

FIGURA 1: Brinquedo terapêutico usado na administração por inalação, Uberaba, MG, Brasil, 2020.

Os dados foram inseridos e armazenados em uma planilha eletrônica do programa Microsoft Office Excel ${ }^{\circledR}$ e importados para o programa estatístico Statistical Package for Social Sciences (SPSS) versão 21.0 para processamento e análise, empregada a estatística descritiva e inferencial. Para avaliar as variações de comportamento antes e após a realização das sessões de BT, foi utilizado o teste de McNemar, considerando um nível de rejeição de 5\% $(p<0,005)$.

O estudo foi registrado no Comitê de Ética em Pesquisa da Universidade Federal do Triângulo Mineiro, assegurando o cumprimento às recomendações da Resolução CNS/MS n466/12, aprovado sob o parecer nำ 1.547.451, identificador CAAE: 51995315.8.0000.5154. 


\section{RESULTADOS}

Foram analisados dados de 99 pré-escolares com idade média de 38 meses de vida, 51\% eram do sexo masculino, $86 \%$ frequentam escola infantil e $90 \%$ estavam acompanhados pela mãe e a tinham como cuidadora principal. Quanto aos agravos de saúde, $74 \%$ tinham passado por internação prévia e, dentre as causas, $28 \%$ foram relacionadas a problemas respiratórios. Todas advinham de causas respiratórias e $74 \%$ eram procedentes da cidade onde está situada a UPA estudada.

Os comportamentos apresentados na Tabela 1 são os sinais observados por crianças que passaram por processos de trauma e dor, representando uma situação de crise. Dos 25 comportamentos avaliados antes e após o uso do BT durante a administração por inalação, três não apresentaram significância estatística ( $p<0,05)$ : “Comportamento protetor", "Não responde a estímulos, demonstrando indiferença" e "Soluço". Contudo, nota-se que sinais físicos como "choro", "grito", "expressão facial de medo", "tensão muscular" foram reduzidos significativamente, como demonstrado na Tabela 1.

TABELA 1. Distribuição de frequências de observação de comportamentos de pré-escolares em uso da administração por inalação, antes e após o uso de do BT. Uberaba, MG, 2020*.

\begin{tabular}{|c|c|c|c|c|c|}
\hline \multirow{2}{*}{ Comportamentos } & \multicolumn{2}{|c|}{ Antes do BTI } & \multicolumn{2}{|c|}{ Após do BTI } & \multirow[t]{2}{*}{$\mathrm{p}$} \\
\hline & $\mathrm{N}$ & $\mathrm{S}(\%)$ & $\mathrm{N}$ & $\mathrm{S}(\%)$ & \\
\hline Colabora passivamente & 18 & 18,2 & 76 & 76,8 & $<0,001$ \\
\hline Comportamento protetor & 21 & 21,2 & 68 & 68,7 & $<0,001$ \\
\hline Permanece calada & 63 & 63,6 & 21 & 21,2 & $<0,001$ \\
\hline Expressão facial de medo & 78 & 78,8 & 38 & 38,4 & $<0,001$ \\
\hline Tensão muscular & 95 & 96,0 & 11 & 11,1 & $<0,001$ \\
\hline Mesmo com a dificuldade de respirar não aceita a colocação do inalador & 97 & 97,9 & 5 & 5,1 & $<0,001$ \\
\hline Balança a cabeça de forma negativa para não ficar com o inalador & 92 & 92,9 & 7 & 7,1 & $<0,001$ \\
\hline Solicita a presença da mãe & 98 & 99,0 & 38 & 38,4 & $<0,001$ \\
\hline Solicita para mãe retirar o inalador & 98 & 99,0 & 24 & 24,2 & $<0,001$ \\
\hline Evita olhar para o profissional e para a inciso & 56 & 56,6 & 35 & 35,4 & $<0,005$ \\
\hline Responde com monossílabos & 96 & 97,0 & 9 & 9,1 & $<0,001$ \\
\hline Chora & 65 & 65,7 & 10 & 10,1 & $<0,001$ \\
\hline Agarra o inalador sobre a face quando percebe melhora do padrão respiratório & 24 & 24,2 & 88 & 88,8 & $<0,001$ \\
\hline Grita & 81 & 81,8 & 5 & 5,1 & $<0,001$ \\
\hline Pede para interromper o procedimento de qualquer jeito & 71 & 71,7 & 19 & 19,2 & $<0,001$ \\
\hline Não responde a estímulos, demonstrando indiferença & 48 & 48,5 & 61 & 61,6 & 0,111 \\
\hline Observa atentamente o profissional que esta instalando a inalação & 49 & 49,5 & 79 & 79,8 & $<0,001$ \\
\hline Verbaliza a melhora do padrão respiratório ao final da terapia inalatória & 95 & 96,0 & 77 & 77,8 & $<0,001$ \\
\hline Postura relaxada - Expressão facial relaxada & 35 & 35,4 & 73 & 73,7 & $<0,001$ \\
\hline Brinca com inalador & 24 & 24,2 & 76 & 76,8 & $<0,001$ \\
\hline Faz pergunta a mãe sobre a terapia inalatória & 48 & 48,5 & 90 & 99,9 & $<0,001$ \\
\hline Faz pergunta ao profissional sobre a terapia inalatória & 50 & 50,5 & 80 & 80,8 & $<0,001$ \\
\hline Soluço & 26 & 26,3 & 10 & 10,1 & 0,007 \\
\hline Ajuda o profissional espontaneamente & 31 & 31,3 & 74 & 74,7 & $<0,001$ \\
\hline Cerra os olhos & 74 & 74,7 & 63 & 63,6 & $<0,001$ \\
\hline
\end{tabular}

*Teste de McNemar, significantes $\mathrm{p}<0,005$

Percebe-se que na segunda administração por inalação, após a sessão do BT, por estarem mais calmas visível pela expressão fácil relaxadas e colaborativas, os pré-escolares receberam melhor a terapia aceitando utilizar a máscara, não balançando a cabeça para retirá-la e a mantiveram no rosto por haver melhora na respiração e chegaram a brincar com o inalador.

A interação com o profissional ficou mais agradável após o uso do BT, fato notado por meio do comportamento da criança em ajudar o profissional espontaneamente (74,7\%) e fazer perguntas para o mesmo (80,8\%). Anteriormente, as mesmas não queriam nem ao menos observá-los (49,5\%) e o brinquedo permitiu que a criança ficasse mais à vontade para observar as atitudes e como o profissional realizava o procedimento (79,8\%). A melhora na comunicação também ficou evidente quando, após o BT apenas 9,1\% dos pré-escolares responderam em monossílabos. 
Percebeu-se que a criança após a sessão ficava mais calma e recebia de forma correta a administração por inalação, sem derramar ou tirar do rosto, resultando em melhor resposta clínica.

\section{DISCUSSÃO}

O uso do BT durante da administração por inalação mostrou-se eficaz para reduzir comportamentos negativos advindos de um procedimento terapêutico.

$\mathrm{O}$ atendimento de enfermagem em unidades de pronto atendimento em sua maioria são procedimentos rápidos e com pouca interação, seja pela curta permanência do usuário ou pela gravidade da situação ${ }^{7}$. Este ambiente para a criança é como estar em contexto perturbador, pois para ela está relacionada com a necessidade de procedimentos invasivos e dolorosos e, dessa forma, a utilização do BT propicia segurança e conforto, após seu uso a criança passa a enxergar o hospital ou a unidade de emergência como um lugar menos cruel $^{1}$.

Comportamentos apresentados na Tabela 1 também foram prevalentes em outros estudos, sendo o choro, o grito, a tensão muscular, a expressão facial de medo manifestações frequentes durantes os procedimentos infantis, sejam eles dolorosos ou não $0^{1,19,21,22}$. Notou-se desfecho positivo em todos os comportamentos específicos a administração por inalação, os pré-escolares receberam melhor a medicação após a sessão do BT por manterem adequadamente o inalador na face.

Estudo analisando o uso do brincar na terapia por aerossóis, evidenciou que apenas 38,9\% de pré-escolares concluíam a administração. Após o uso de uma técnica lúdica visual a adesão subiu para $92,9 \%{ }^{12}$. Demonstrando a importância do brincar mesmo em situações de doenças agudas, o lúdico auxilia a compressão dentro do universo da criança, fazendo com que o procedimento não seja encarado como algo que irá lhe fazer mal ou causar dor. Na administração por inalação o uso da máscara e o barulho do aparelho ou rede de oxigênio causam medo e ansiedade por acharem que serão submetidas a procedimentos invasivos. Isto associado ao desconforto da própria patologia pioram a adesão ${ }^{10-12}$.

A interação e a comunicação com o profissional são prejudicadas em situações de medo e dor, a criança se comunica por meio de monossílabas não permitindo a realização do procedimento. Com a inserção do BT, nota-se neste e em outros estudos ${ }^{1,6,18}$ que as crianças passam a colaborar com os profissionais, a observá-los, fazem perguntas, deixam de se comunicar por curtas respostas e não interrompem o procedimento com tanta frequência.

A presença da mãe traz conforto e segurança para as crianças em situações desagradáveis, de forma que a maioria solicita a companhia materna ${ }^{1,19,23}$. Os profissionais devem enxergar a mãe e a família como fonte de apoio à criança e incluí-los nos cuidados e nas sessões do BT faz com que a criança fique mais tranquila e participativa ${ }^{1}$.

Os materiais para construção do BT são considerados de baixo custo e acessíveis, sua aplicação é fácil e possui boa aceitação por parte das crianças, capazes de transformar os ambientes, criando um clima descontraído e mais próximo do contexto da criança ${ }^{2,3}$. Na prática observa-se baixa adesão pelos profissionais de enfermagem, relatam a falta de tempo e de capacitação profissional como entraves ${ }^{24}$.

Eentre as limitações do estudo, destacaram-se o tempo demandado a coleta de dados para atingir o período de sazonalidade da doença respiratória na região e a divulgação da realidade de um único cenário, o que, em contrapartida, é superado pelos achados convergentes com publicações sobre o tema.

\section{CONCLUSÃO}

Nos pré-escolares participantes do estudo foram registradas reações que otimizaram os comportamentos, evidenciando maior aceitação e adaptação à administração por inalação. O número reduzido de comportamentos ou situações de estresse entre os pré-escolares, como chorar, agitar-se, debater-se ou gritar, indica os efeitos benéficos do BT para esse procedimento, reforçando a importância da aplicação do BT na assistência de enfermagem pediátrica.

Assim, os resultados sinalizam a importância em implementar o uso do BT dentro nas unidades de saúde, especialmente em serviços de pronto atendimento pediátrico sempre que a criança paciente apresentar dificuldade em compreender a situação diante da administração por inalação.

\section{REFERÊNCIAS}

1. Caleffi CCF, Rocha PK, Anders JC, Souza AIJ, Burciaga VB, Serapião LS. Contribution of structured therapeutic play in a nursing care model for hospitalised children. Rev. Gaúcha Enferm. 2016; 37(2):e58131. DOI: http://dx.doi.org/10.1590/19831447.2016.02.58131

2. Freitas BHBM, Voltani SSAA. Therapeutic play in the pediatric urgent and emergency department: an integrative literature review. Cogitare Enferm. 2016; 21(1):1-8. DOI: http://dx.doi.org/10.5380/ce.v21i4.40728 
3. Silva SGT, Santos MA, Floriano CMF, Damião EBC, Campos FV, Rossato LM. Influence of Therapeutic Play on the anxiety of hospitalized school-age children: clinical trial. Rev. Bras. Enferm. 2017; 70(6):1244-9. DOI: http://dx.doi.org/10.1590/0034-71672016-0353

4. Sabino AS, Esteves AVF, Oliveira APP, Silva MVG. The parents' knowledge on the care process through play. Cogitare Enferm. 2018 [cited 2020 Jun 29]; 23(2):e52849. Available from: https://revistas.ufpr.br/cogitare/article/view/52849/pdf

5. Barroso MCCS, Santos RSFV, Santos AEV, Nunes MDR, Lucas EAJCF. Children's perception of venipuncture through therapeutic toy. Acta paul. enferm. 2020; 33:e-APE20180296. DOI: https://doi.org/10.37689/acta-ape/2020ao0296

6. Silva JRS, Pizzoli LML, Amorim ARP, Pinheiros FT, Romanini GC, Silva JG, et al. Using therapeutic toys to facilitate venipuncture procedure in preschool children. Pediatr. Nurs. [Internet]. 2016 [cited 2020 Fev 26]; 42(2):61-8. Avaliable from: https://www.pediatricnursing.net/ce/2018/article42026168.pdf

7. Berté C, Ogradowski KRP, Zagonel IPS, Tonin L, Favero L, Almeida Junior RDL. Therapeutic toy in the context of pediatric emergency. Rev. Baiana Enferm. 2017; 31(3):e20378. Available from: https://portalseer.ufba.br/index.php/enfermagem/article/download/20378/15160

8. Prato MIC, Silveira A, Neves ET, Buboltz FL. Respiratory diseases in childhood: an integrative review. Rev. Soc. Bras. Enferm. Ped. [Internet]. 2014 [cited 2019 Aug 26]; 14(1):33-9. Available from: https://sobep.org.br/revista/images/stories/pdf-revista/vol14n1/v14_n1_artigo_revisao_1.pdf

9. Velasco HF, Cabral CZ, Pinheiro PP, Azambuja RCS, Vitola LS, Costa MR, et al. Use of digital media for the education of health professionals in the treatment of childhood asthma. J. Pediatr. (Rio J.). 2015 [cited 2019 Aug 26]; 91(2):183-8. DOI: http://dx.doi.org/10.1016/j.jped.2014.07.007

10. Trivedi D. Interventions to improve inhaler technique for people with asthma. Prim. Health Care Res. Dev. 2019 [cited 2019 Nov 26]; 20(e72):1-2. DOI: https://doi.org/10.1017/S1463423619000501

11. Padding, AM, Rutjes, NW, Hashimoto, S. et al. Young children experience little emotional burden during invasive procedures in asthma research. Eur. J. Pediatr. 2019 [cited 2019 Nov 26]; 178:207-211. DOI: https://doi.org/10.1007/s00431-018-3265-0

12. Hsiu-jung C, Yu-chin H, Yi-Fang H, Yen-Yi C. Therapeutic play promoting children health management-preschool children aerosol therapy completion rates. IJRMBS [Internet] 2014 [cited 2020 Fev 26]; 88(1). Available from: http://ijrmbs.com/vol1issue1/2/yuchin.pdf

13. Wegner W, Silva MUM, Peres MA, Bandeira LE, Frantz E, Botene DZA, et al. Patient safety in the care of hospitalised children: evidence for pediatric nursing. Rev. Gaúcha Enferm. 2017 [cited 2019 Nov 26]; 38(1):e68020. DOI: https://doi.org/10.1590/19831447.2017.01.68020

14. Eccleston C, Fisher E, Law E, Bartlett J, Palermo TM. Psychological interventions for parents of children and adolescents with chronic illness. Cochrane Database Syst. Rev. 2015 [cited 2019 Nov 26]; 4:CD009660. Available from: https://doi.org/10.1002/14651858.CD009660.pub3

15. Moreira TR, Bandeira STA, Lopes SC, Carvalho SL, Negreiros FDS, Neves CS. Difficulties concerning Diabetes Mellitus Type 1 in children and adolescents. Rev. Rene. 2016 [cited 2019 Apr 10]; 17(5):651-8. Available from: http://periodicos.ufc.br/rene/article/view/6196/4432

16. Lemos I, Oliveira J, Gomes E, Silva K, Silva P, Fernandes G. Brinquedo terapêutico no procedimento de punção venosa: estratégia para reduzir alterações comportamentais. Rev. Cuid. 2016 [cited 2019 Apr 10]; 7(1): 1163-70. DOI: http://dx.doi.org/10.15649/cuidarte.v7i1.303

17. Paladino CM, Carvalho R, Almeida FA. Therapeutic play in preparing for surgery: behavior of preschool children during the perioperative period. Rev. Esc. Enferm. USP. 2014 [cited 2019 Apr 10]; 48(3):423-429. DOI: https://doi.org/10.1590/S0080623420140000300006

18. Pontes JED, Tabet E, Folkmann MAS, Cunha MLR, Almeida FA. Therapeutic play: preparing the child for the vaccine. Einstein (São Paulo) [online]. 2015 [cited 2019 Apr 10]; 13(2):238-242. DOI: https://doi.org/10.1590/S1679-45082015AO2967

19. Guimarães MVR, Teixeira ER. Family care for infants with respiratory diseases: an exploratory descriptive study. Online braz. j. nurs. 2015 [cited 2019 Nov 10]; 14(3):313-23. DOI: https://doi.org/10.17665/1676-4285.20155210

20. Polit DF, Beck CT, Hungler BP. Fundamentos de pesquisa em enfermagem: métodos, avaliação e utilização. 9. ed. Porto Alegre: ArtMed; 2019.

21. Pennafort VPS, Queiroz MVO, Gomes ILV, Rocha MFF. Instructional therapeutic toy in the culture care of the child with diabetes type 1. Rev. Bras. Enferm. 2018 [cited 2019 Apr 10]; 71(3):1334-1342. DOI: https://doi.org/10.1590/0034-7167-2017-0260

22. Lemos ICS, Oliveira JD, Gomes EB, Silva KVL, Silva PKS, Fernandes GP. Brinquedo terapêutico no procedimento de punção venosa: estratégia para reduzir alterações comportamentais. Rev. Cuid. (Bucaramanga. 2010). 2016; 7(1):1163-70. DOI: http://dx.doi.org/10.15649/cuidarte.v7i1.303

23. Fontes CMB, Oliveira ASS, Toso LA. Therapeutic toy in pediatric intensive therapy unit. Rev. enferm. UFPE on line. 2017 [cited 2019 Nov 10]; 11(supl.7):2907-2915. Available from: https://periodicos.ufpe.br/revistas/revistaenfermagem/article/view/9518

24. Marques EP, Garcia TMB, Anders JC, Luz JH, Rocha PK, Souza S. Playful activities in healthcare for children and adolescents with cancer: the perspectives of the nursing staff. Esc. Anna Nery Rev. Enferm. 2016 [cited 2019 Nov 10]; 20(3): e20160073. Available from: http://www.scielo.br/scielo.php?script=sci_arttext\&pid=S1414-81452016000300218\&Ing=en 